\title{
Ionic separation efficiency of a novel electric-field-assisted membrane module comprising an array of microchannel units
}

\author{
Jyh-Ping Hsu ${ }^{\text {a }}$, Sung-Hwa Lin ${ }^{\mathrm{b}, *}$, Shiojenn Tseng ${ }^{\mathrm{c}}$, Ay Su ${ }^{\mathrm{d}}$, Duu-Jong Lee ${ }^{\mathrm{a}}$, Chur-Jen Chen ${ }^{\mathrm{e}}$ \\ ${ }^{a}$ Department of Chemical Engineering, National Taiwan University, Taipei 10617, Taiwan \\ ${ }^{\mathrm{b}}$ Department of Chemical Engineering and Materials Engineering, National Ilan University, Yilan 26047, Taiwan \\ ${ }^{\mathrm{c}}$ Department of Mathematics, Tamkang University, Taipei 25137, Taiwan \\ ${ }^{\mathrm{d}}$ Department of Mechanical Engineering \& Fuel Cells Research Center, Yuan Ze University, Taoyuan 32003, Taiwan \\ e Department of Mathematics, Tunghai University, Taichung 40704, Taiwan
}

Received 10 October 2006; accepted 29 November 2006

Available online 3 January 2007

\begin{abstract}
The ionic separation efficiency of a novel membrane module comprising an array of microchannel units is analyzed. Under the Debye-Hückel approximation, we derive a semianalytical expression for the ionic separation efficiency. Analyses reveal that the effects of the size of the microchannel, the fixed charge density in the membrane layer, and the permittivity of the membrane layer on ionic separation efficiency depend strongly on the valence type of electrolyte in treated water. Under the condition of a symmetric electrolyte, the ionic separation efficiency is found to be about unity and unresponsive to variation of system parameters. If the valence of the cation is higher than that of the anion, the ionic separation efficiency is larger than unity, and decreases to unity as the size of the microchannel increases. In contrast, if the valence of the cation is lower than that of the anion, the ionic separation efficiency is smaller than unity and increases to unity as the size of the microchannel increases. Under the latter two conditions, the effects of both fixed charge density in the membrane layer and permittivity of the membrane layer on the ionic separation efficiency are found to be reversed.
\end{abstract}

(C) 2006 Elsevier Inc. All rights reserved.

Keywords: Microchannel unit; Charged membrane layer; Ionic separation efficiency; Debye-Hückel approximation; Valence type of electrolyte

\section{Introduction}

In water treatment, electric-field-assisted ion-selective membranes are used to reduce the content of toxic ions or unallowable charged materials in wastewater. It is known that, with an applied electric field, the presence of an electrical double layer near a charged surface is capable of improving the ionic separation efficiency of a solution containing electrolytes [1-12]. Under typical conditions, the thickness of an electrical double layer ranges from a few nanometers to a few micrometers. This implies that the linear size of a device appropriate to assessing the effect of an electrical double layer ranges from submicrometers to micrometers [13-25]. The structure of an electrical double layer is influenced mainly by the charged conditions over a

\footnotetext{
* Corresponding author.

E-mail address: shlin@niu.edu.tw (S.-H. Lin).
}

rigid surface (or inside a membrane layer in the case of soft surfaces) and the nature of the electrolytes in the liquid phase. The former include the sign and the density of the charge over a rigid surface (or inside a membrane layer), the corresponding charge distribution, and the origin of the charge, such as the dissociation/association reactions of functional groups. The latter includes the ionic strength of the electrolyte solution and the valences of the ionic species. Depending upon the problem considered and the conditions assumed, the structure of an electrical double layer may be dominated by one of these two factors. Apparently, the roles these factors play in the assessment of a microsized device deserve thorough study.

In previous work [26], we proposed a membrane module of an array of microchannel units. For systematic purposes, cylindrical macrochannels of unified geometry are collocated uniformly over the charged membrane in such a way that all the microchannels are aligned in the axial direction parallel to 
the transversal of the membrane, that is, the direction of the effluent. Based on the Debye-Hückel approximation, the onedimensional electrokinetic flow under an axial electric field is solved, and we arrive at analytical solutions for the electric field in the electrical double layer, for the velocity field of an electrolyte solution, and for the effluent flow rate. The effluent flow rate relative to that driven by a hydrodynamic pressure drop is found to increase with the charge density in the membrane layer, the permittivity of the membrane layer, and the applied electric field. The results also reveal that an optimal scale of microchannel exists for the effluent flow rate. The present study focuses on the ionic separation efficiency of the membrane module. In particular, the influences of the fixed charge density and the permittivity of the membrane layer and of the valences of the ionic species in the liquid phase on the separation efficiency are discussed. For analysis, the fixed charge in membrane layer is confined to be uniform and constant, and to be negative in sign. When the electrical double layer is absent, the flow of filtrate is zero for a neutral electrolyte solution. Due to the applied electric field, however, the ions in the electrolyte solution are separated. Hence, to assess the net effect of an electrical double layer on ionic separation, the relevant analyses are conducted on the basis of ionic separation efficiency for the case when the electrical double layer is absent.

\section{Theory}

The proposed membrane module was elaborated in our previous work [26], where we arrived at the solutions of both electrical potential field and velocity field in a microchannel unit analytically. For homogeneity of the membrane module, only a single microchannel unit is considered. As Fig. 1 demonstrates, the radial and axial coordinates are, respectively, $r$ and $z$, and $r$ originates from the radial center of the microchannel unit. The microchannel unit is $r_{\mathrm{mcu}}$ in radius, and the microchannel is $r_{\mathrm{mc}}$ in radius. A uniform, axial electric field of magnitude $E_{z}$ is applied in the direction of positive $z$. As in the previous analysis, we assume that

(1) The diffusion of ionic species across the membrane layer is negligible.

(2) The end effect is negligible.

(3) The distortion of the electrical double layer is negligible.

(4) The Debye-Hückel condition is satisfied.

(5) The resistance to the flow of liquid in the membrane layer is sufficiently large so that the flow of liquid inside is negligible.

(6) The liquid phase is a Newtonian fluid of constant physical properties, and the flow field is in the creeping flow regime.

When the electric field is applied, cations and anions in the solution move in opposite directions; hence ions of different signs are separated. Since the surface membrane is negatively charged, the solution in the microchannel flows along the direction of positive $z$, due to richer cations. Conventionally, the efficiency of ionic separation is measured by the magnitude of electric current in the microchannel. The movements

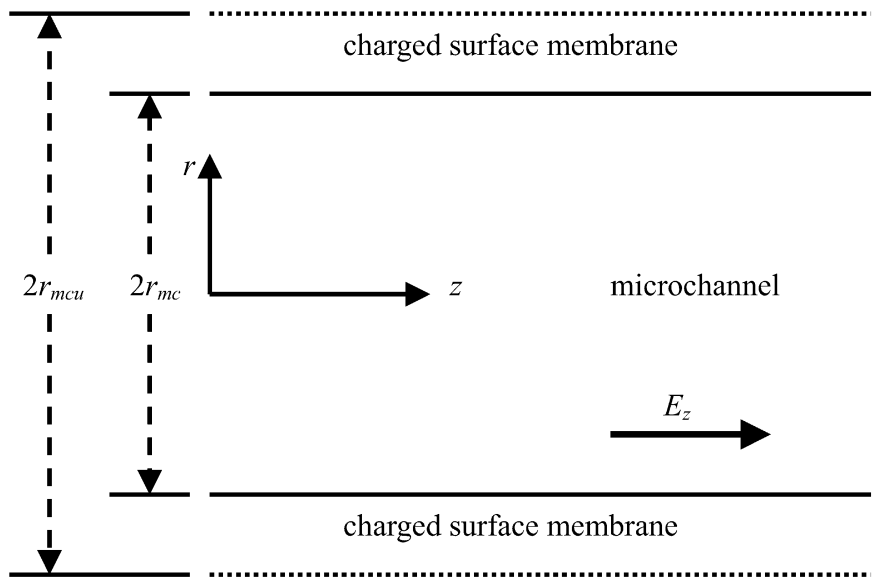

Fig. 1. Schematic representation of a microchannel unit.

of both cations and anions contribute to the electric current. In the microchannel, the electrical double layer arises from the charged surface membrane. The presence of the electrical double layer may elevate/depress the ionic separation, depending on the structure of the electrical double layer. From the definition of the electric current density, $i_{z}$, we have

$$
\begin{aligned}
i_{z} & =\sum_{i=1}^{n} z_{i} F c_{i} u_{i}=\sum_{i=1}^{n} z_{i} F c_{i}^{0} e^{-z_{i} F \phi / R_{\mathrm{gc}} T}\left(u_{z}+m z_{i} F E_{z} / N_{\mathrm{A}}\right) \\
& \cong \sum_{i=1}^{n} z_{i} F c_{i}^{0}\left(1-z_{i} F \phi / R_{\mathrm{gc}} T\right)\left(u_{z}+m z_{i} F E_{z} / N_{\mathrm{A}}\right)
\end{aligned}
$$

where $z_{i}, c_{i}$, and $c_{i}^{0}$ are, respectively, the valence, the molar concentration in the diffuse layer, and the molar concentration in the bulk solution for mobile ion $i ; u_{i}, u_{z}$, and $m$ are, respectively, the axial velocity of mobile ion $i$, the axial velocity of the electrolyte solution in the microchannel, and the mobility of mobile ions; $\phi, n$, and $T$ are, respectively, the electrical potential due to the electrical double layer, the number of kinds of ions in the electrolyte solution, and the absolute temperature of the system; and $F, R_{\mathrm{gc}}$, and $N_{\mathrm{A}}$ are, respectively, the Faraday constant, the gas constant, and Avogadro's number. Note that the velocity of mobile ions comprises two parts, that of the electrolyte solution and that of mobile ions relative to the electrolyte solution. The latter is proportional to the product of the mobility of the mobile ions and the electric force due to the applied electric field. In general, the mobility of mobile ions might depend on their mass, their shape, and the charge they carry. For simplicity, we assume that it is a constant, $m$. The radial dependence of $\phi$ and $u_{z}$ is derived in our previous work [26]. By the Debye-Hückel approximation, the highly nonlinear terms (exponential terms) are reduced to linear ones, as expressed in the second line in Eq. (1). Integrating the electric current density over the radial cross section of the microchannel, the electric current in the microchannel, $I_{\mathrm{ec}}$, can be restored. That is,

$I_{\mathrm{ec}}=2 \pi \int_{0}^{r_{\mathrm{mc}}} r i_{z} \mathrm{~d} r$. 
From the neutrality in bulk solution we have

$\sum_{i=1}^{n} z_{i} c_{i}^{0}=0$

Substituting Eq. (1) into Eq. (2), with the aid of Eq. (3), and Eqs. (8) and (20) in previous work [26], we arrive at the expression for the scaled electric current, $I^{*}$, as

$$
\begin{aligned}
I^{*}= & \frac{I_{\mathrm{ec}}}{I_{\mathrm{ec}}^{0}}=\frac{I_{\mathrm{ec}}}{\pi r_{\mathrm{mc}}^{2} 2 I F^{2} m E_{z} / N_{\mathrm{A}}} \\
= & 1+\frac{1}{m^{*}} \frac{1}{R_{\mathrm{mc}}} A_{1}^{2} I_{0}\left(R_{\mathrm{mc}}\right) I_{1}\left(R_{\mathrm{mc}}\right) \\
& -\frac{1}{m^{*}} \frac{1}{R_{\mathrm{mc}}^{2}} A_{1}^{2} \int_{0}^{R_{\mathrm{mc}}} R I_{0}^{2}(R) \mathrm{d} R \\
& -\frac{\sum_{i=1}^{n} z_{i}^{3} c_{i}^{0}}{I} \frac{1}{R_{\mathrm{mc}}} A_{1} I_{1}\left(R_{\mathrm{mc}}\right),
\end{aligned}
$$

where $I_{0}$ and $I_{1}$ are, respectively, the modified Bessel functions of the first kind of orders 0 and $1, I$ is the ionic strength of the electrolyte solution, $R=\kappa r$, with $\kappa$ being the Debye-Hückel parameter defined by $\kappa=\left(2 I F^{2} / \varepsilon_{\mathrm{mc}} R_{\mathrm{gc}} T\right)^{1 / 2}$, where $\varepsilon_{\mathrm{mc}}$ is the permittivity of the electrolyte solution, $R_{\mathrm{mc}}=\kappa r_{\mathrm{mc}}$, and $m^{*}$ is defined by

$m^{*}=\frac{F^{2} \mu m}{2 \varepsilon_{\mathrm{mc}} R_{\mathrm{gc}} T N_{\mathrm{A}}}$,

where $\mu$ is the viscosity of the electrolyte solution. The dimensionless coefficient in Eq. (4), $A_{1}$, is a function of $\rho_{\mathrm{ef}}^{*}=$ $\rho_{\mathrm{ef}} / 2 I F$ with $\rho_{\text {ef }}$ being the fixed charge density in the membrane, $\varepsilon^{*}=\varepsilon_{\mathrm{mcs}} / \varepsilon_{\mathrm{mc}}$ with $\varepsilon_{\mathrm{mcs}}$ being the permittivity in the membrane layer, $R_{\mathrm{mc}}$, and $R_{\mathrm{mcu}}=\kappa r_{\mathrm{mcu}}$. The value of $A_{1}$ is evaluated by the equations

$$
\begin{aligned}
A_{1}= & \Delta_{A_{1}} / \Delta, \\
\Delta= & I_{0}\left(R_{\mathrm{mc}}\right)\left[I_{1}\left(\varepsilon^{*-1 / 2} R_{\mathrm{mc}}\right) K_{1}\left(\varepsilon^{*-1 / 2} R_{\mathrm{mcu}}\right)\right. \\
& \left.-I_{1}\left(\varepsilon^{*-1 / 2} R_{\mathrm{mcu}}\right) K_{1}\left(\varepsilon^{*-1 / 2} R_{\mathrm{mc}}\right)\right] \\
& -\varepsilon^{*-1 / 2} I_{1}\left(R_{\mathrm{mc}}\right)\left[I_{0}\left(\varepsilon^{*-1 / 2} R_{\mathrm{mc}}\right) K_{1}\left(\varepsilon^{*-1 / 2} R_{\mathrm{mcu}}\right)\right. \\
+ & \left.I_{1}\left(\varepsilon^{*-1 / 2} R_{\mathrm{mcu}}\right) K_{0}\left(\varepsilon^{*-1 / 2} R_{\mathrm{mc}}\right)\right], \\
\Delta_{A_{1}}= & \rho_{\mathrm{ef}}^{*}\left[I_{1}\left(\varepsilon^{*-1 / 2} R_{\mathrm{mc}}\right) K_{1}\left(\varepsilon^{*-1 / 2} R_{\mathrm{mcu}}\right)\right. \\
& \left.-I_{1}\left(\varepsilon^{*-1 / 2} R_{\mathrm{mcu}}\right) K_{1}\left(\varepsilon^{*-1 / 2} R_{\mathrm{mc}}\right)\right],
\end{aligned}
$$

where $I_{0}$ and $I_{1}$ are, respectively, the modified Bessel functions of the first kind of order 0 and 1 , and $K_{0}$ and $K_{1}$ are, respectively, the modified Bessel functions of the second kind of order 0 and 1. In Eq. (4) the scaling factor $I_{\mathrm{ec}}^{0}$, the electric current when the electrical double layer is absent, is introduced to measure the influence of the electrical double layer on the electric current. If the electrical double layer is absent, both $u_{z}$ and $\phi$ vanish under an axial electric field. Therefore, $I_{\mathrm{ec}}^{0}$ can be deduced directly from Eqs. (1) and (2) as

$I_{\mathrm{ec}}^{0}=\pi r_{\mathrm{mc}}^{2} 2 I F^{2} m E_{z} / N_{\mathrm{A}}$.

\section{Results and discussion}

Supposing that the liquid phase contains $z_{1}: z_{2}$ electrolyte only, Eq. (3) is simplified into

$z_{1} c_{1}^{0}+z_{2} c_{2}^{0}=0$,

where $c_{1}^{0}$ and $c_{2}^{0}$ are, respectively, the molar concentration of the cation and that of the anion. By means of Eq. (10), we have the following relations:

$$
\begin{aligned}
& I=\frac{1}{2}\left(z_{1}^{2} c_{1}^{0}+z_{2}^{2} c_{2}^{0}\right) \\
&=\frac{1}{2} z_{1} c_{1}^{0}\left(z_{1}-z_{2}\right)=\frac{1}{2} z_{2} c_{2}^{0}\left(-z_{1}+z_{2}\right), \\
& c_{1}^{0}=\frac{2 I}{z_{1}\left(z_{1}-z_{2}\right)}, \\
& c_{2}^{0}=\frac{2 I}{z_{2}\left(-z_{1}+z_{2}\right)}, \\
& z_{1}^{3} c_{1}^{0}+z_{2}^{3} c_{2}^{0}=2 I\left(z_{1}+z_{2}\right) .
\end{aligned}
$$

When Eq. (14) is substituted into Eq. (4), Eq. (4) becomes

$$
\begin{aligned}
I^{*}=1 & +\frac{1}{m^{*}} \frac{1}{R_{\mathrm{mc}}} A_{1}^{2} I_{0}\left(R_{\mathrm{mc}}\right) I_{1}\left(R_{\mathrm{mc}}\right) \\
& -\frac{1}{m^{*}} \frac{1}{R_{\mathrm{mc}}^{2}} A_{1}^{2} \int_{0}^{R_{\mathrm{mc}}} R I_{0}^{2}(R) \mathrm{d} R \\
& -2\left(z_{1}+z_{2}\right) \frac{1}{R_{\mathrm{mc}}} A_{1} I_{1}\left(R_{\mathrm{mc}}\right) .
\end{aligned}
$$

The expression in Eq. (15) implies that $I^{*}$ is a function of $A_{1}$, $R_{\mathrm{mc}}$, and $z_{1}+z_{2}$. Referring to Eqs. (6)-(8), the coefficient $A_{1}$ is related to $\rho_{\mathrm{ef}}^{*}, \varepsilon^{*}, R_{\mathrm{mc}}$, and $R_{\mathrm{mcu}}$. The value of $R_{\mathrm{mcu}}$ is fixed in subsequent discussion. Therefore, $I^{*}$ is mainly dependent on $\rho_{\mathrm{ef}}^{*}, \varepsilon^{*}, R_{\mathrm{mc}}$, and $z_{1}+z_{2}$. Here, it is interesting to note that $I^{*}$ is independent of $E_{z}$. This is because in the absence of the electrical double layer $I_{\mathrm{ec}}^{0}$ is linearly dependent on $E_{z}$, as described in Eq. (9). On the other hand, when it is present, $u_{z}$ depends linearly on $E_{z}$, as mentioned in our previous work [26], but $\phi$ is independent of $E_{z}$ since the distortion of the electrical double layer arising from $E_{z}$ is neglected. Equations (1) and (2) thus imply that the electric current in the presence of an electrical double layer is also dependent linearly on $E_{z}$. As a consequence, $I^{*}$ is independent of $E_{z}$, which is consistent with Eqs. (4) and (15).

Numerical simulations were conducted to examine the influences of the key parameters of the present problem on the ionic separation efficiency of a microchannel. For illustration we assume that $I=10^{-3} \mathrm{M}$ and $R_{\mathrm{mcu}}=10$, and therefore $\kappa^{-1}=10 \mathrm{~nm}=10^{-8} \mathrm{~m}$ and $r_{\mathrm{mcu}}=100 \mathrm{~nm}=10^{-7} \mathrm{~m}$. Also $\mu=10^{-3} \mathrm{~kg} \mathrm{~m}^{-1} \mathrm{~s}^{-1}$ and $m^{*}=10$. The membrane is negatively charged, $\varepsilon^{*}$ ranges from 0 to $1, \rho_{\mathrm{ef}}^{*}$ ranges from -1 to 0 , and $\left|z_{i}\right|$ is 1,2 , and 3. Equation (15) implies that $z_{1}+z_{2}$ is the determinant; therefore we have five kinds of valence type for the electrolyte, namely (a) $\left(z_{1}, z_{2}\right)=(1,-1),(2,-2)$, and $(3,-3)$, (b) $\left(z_{1}, z_{2}\right)=(2,-1)$ and $(3,-2)$, (c) $\left(z_{1}, z_{2}\right)=$ $(3,-1),(\mathrm{d})\left(z_{1}, z_{2}\right)=(1,-2)$ and $(2,-3)$, and (e) $\left(z_{1}, z_{2}\right)=$ 


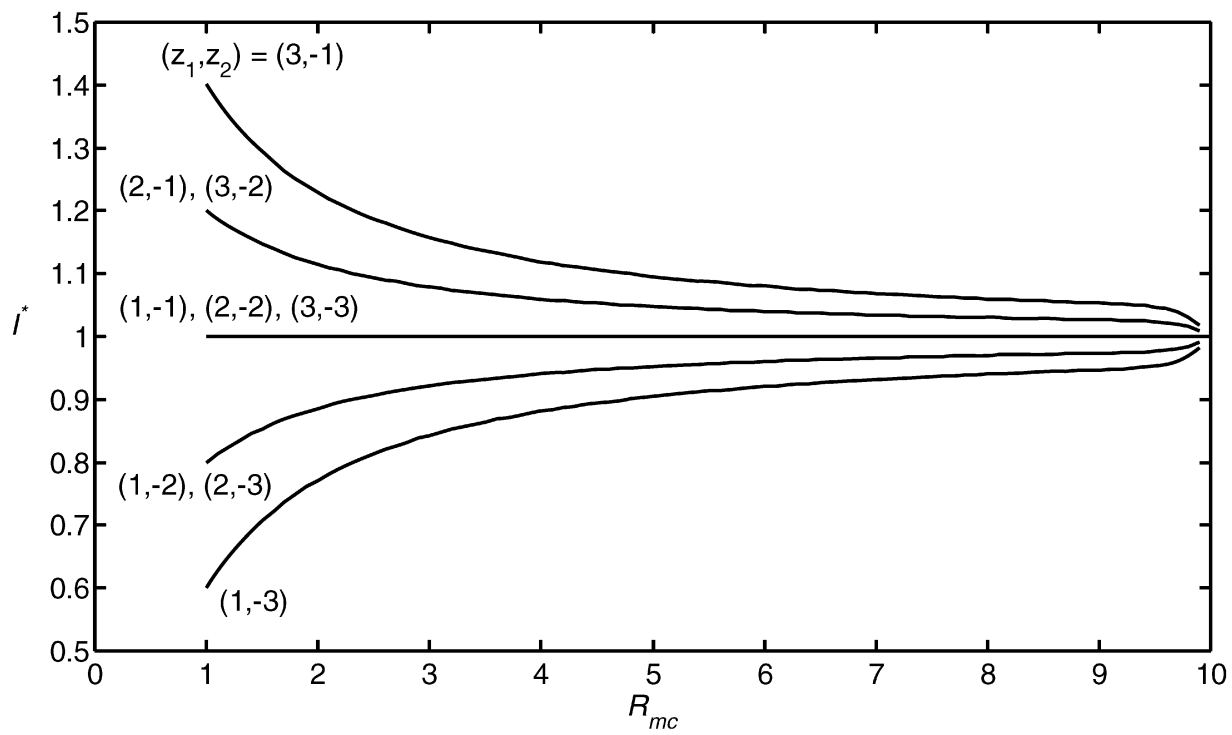

Fig. 2. Variation of $I^{*}$ as a function of $R_{\mathrm{mc}}$ for different values of $\left(z_{1}, z_{2}\right) \cdot \rho_{\mathrm{ef}}^{*}=-0.5 ; \varepsilon^{*}=0.1$.

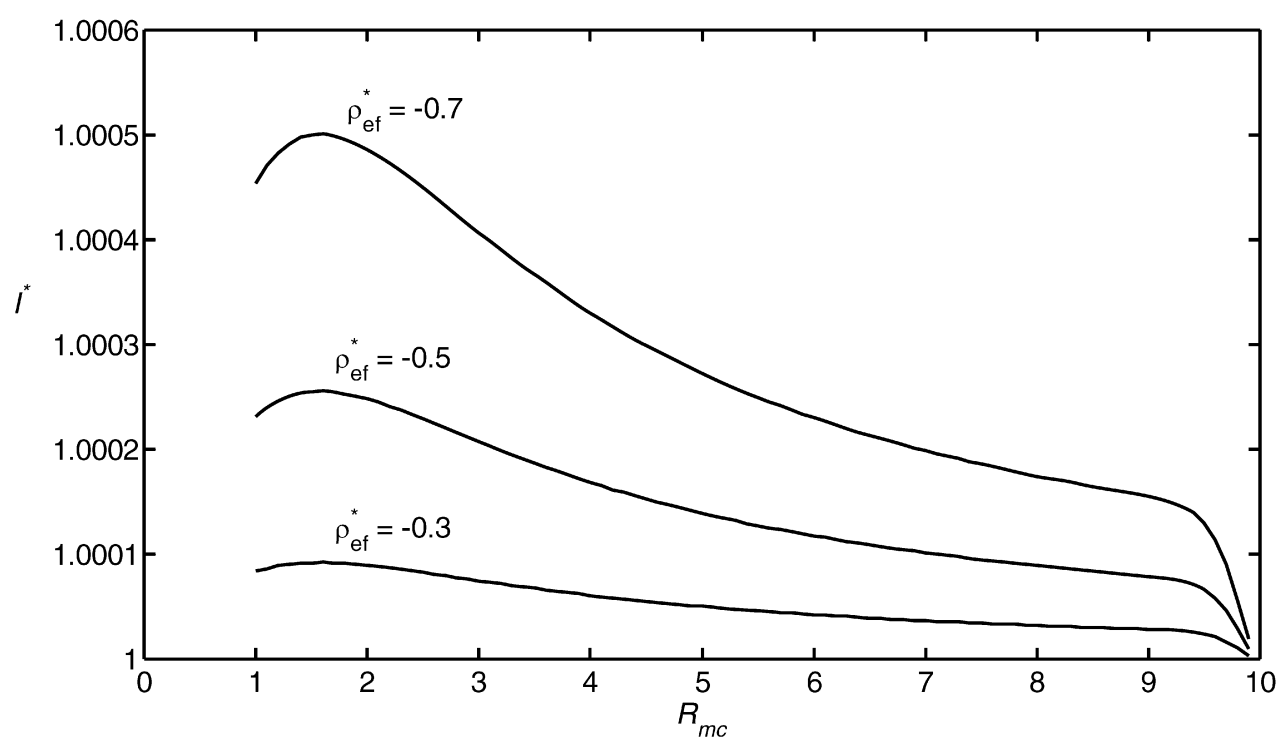

Fig. 3. Variation of $I^{*}$ as a function of $R_{\mathrm{mc}}$ when $\rho_{\mathrm{ef}}^{*}$ changes. $\left(z_{1}, z_{2}\right)=(1,-1),(2,-2)$, and $(3,-3) . \varepsilon^{*}=0.1$.

$(1,-3)$. In (a), the electrolyte is symmetric, or $z_{1}+z_{2}=0$. In (b) and (c), the valence of the cation is higher than that of the anion, or $z_{1}+z_{2}>0$. And in (d) and (e), the valence of the cation is lower than that of the anion, or $z_{1}+z_{2}<0$.

The simulated variations in $I^{*}$ as a function of $R_{\mathrm{mc}}$ for various $\left(z_{1}, z_{2}\right), \rho_{\mathrm{ef}}^{*}$, and $\varepsilon^{*}$ are summarized Figs. $2-8$. The dramatic variation in $I^{*}$ as the type of electrolyte varies shown in Fig. 2 is attributed to the structure of the electrical double layer. The movements of cations and anions are opposite in direction, and both of them contribute to the electric current. Hence, referring to Eq. (1), the increment of electric current depends on both the concentration and the valence of cations and anions. The membrane layer in the microchannel unit is negatively charged; hence the molar concentrations of cations and anions are elevated and depressed, respectively, if compared with those in the bulk electrolyte solution. Due to the Debye-Hückel approxi- mation, the total concentration of mobile ions in the electrical double layer will remain the same as that in bulk solution. This is shown by a mathematical expression as

$$
\begin{aligned}
\sum_{i=1}^{n} c_{i} & =\sum_{i=1}^{n} c_{i}^{0}\left(1-z_{i} F \phi / R_{\mathrm{gc}} T\right) \\
& =\sum_{i=1}^{n} c_{i}^{0}-\left(\sum_{i=1}^{n} z_{i} c_{i}^{0}\right) F \phi / R_{\mathrm{gc}} T=\sum_{i=1}^{n} c_{i}^{0} .
\end{aligned}
$$

Namely, variations of molar concentrations of cations and anions will compensate for each other. Consequently, $I^{*}$ is determined by the molar concentration of the ion with higher valence. We conclude that increasing/decreasing the molar concentration of the ion with higher valence increases/decreases the scaled electric current. By increasing the strength of the electrical double layer, the molar concentrations of cations 


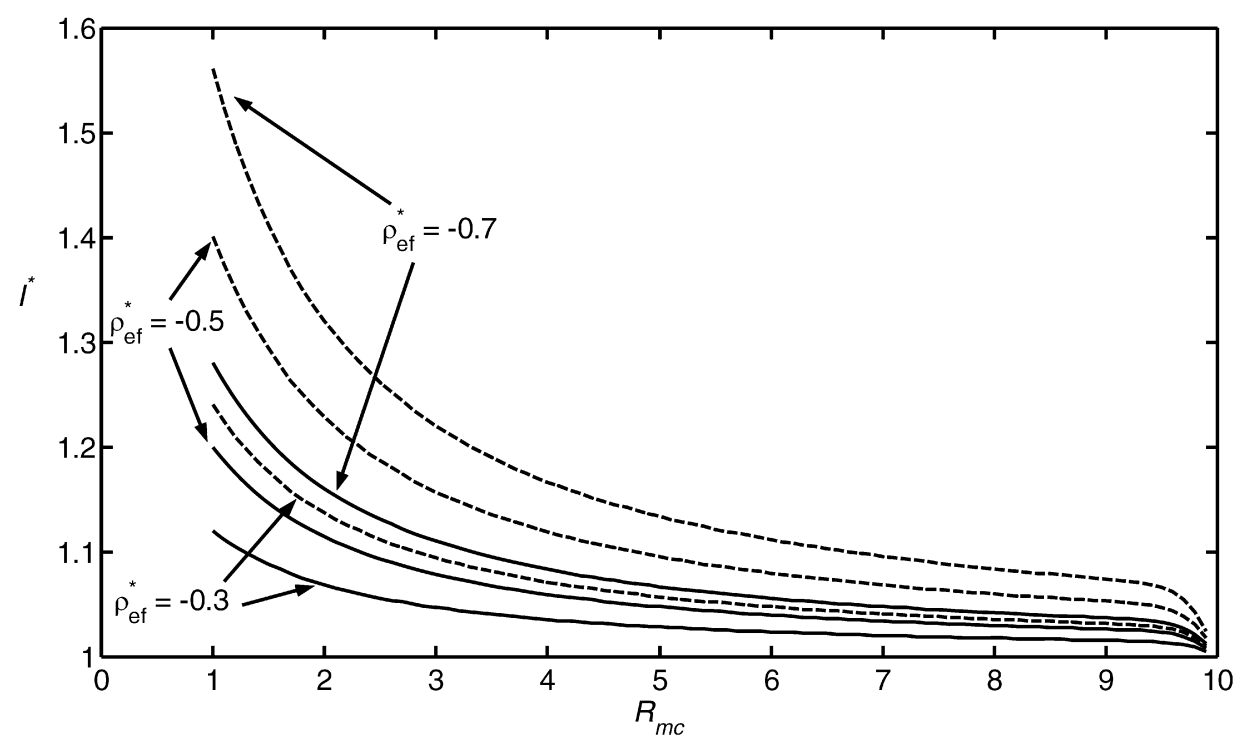

Fig. 4. Variation of $I^{*}$ as a function of $R_{\mathrm{mc}}$ when $\rho_{\mathrm{ef}}^{*}$ changes. Solid curves: $\left(z_{1}, z_{2}\right)=(2,-1)$ and $(3,-2)$. Dashed curves: $\left(z_{1}, z_{2}\right)=(3,-1) \cdot \varepsilon^{*}=0.1$.

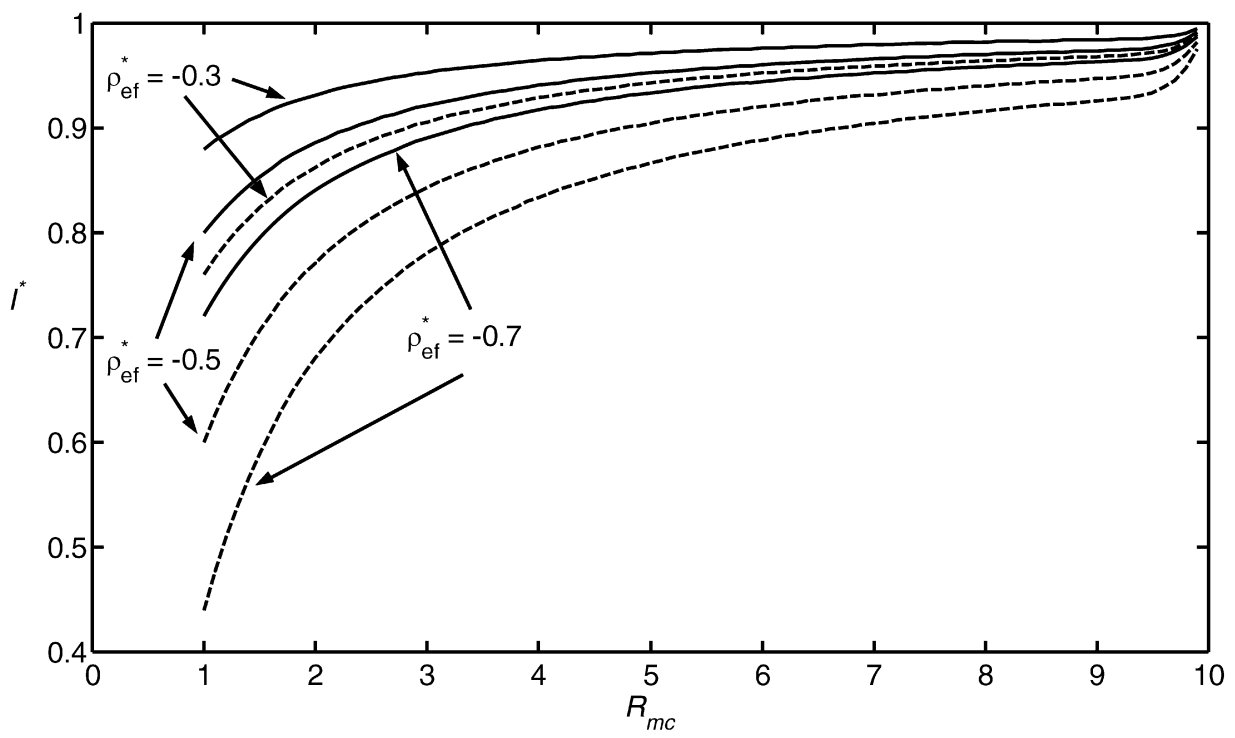

Fig. 5. Variation of $I^{*}$ as a function of $R_{\mathrm{mc}}$ when $\rho_{\mathrm{ef}}^{*}$ changes. Solid curves: $\left(z_{1}, z_{2}\right)=(1,-2)$ and $(2,-3)$. Dashed curves: $\left(z_{1}, z_{2}\right)=(1,-3) \cdot \varepsilon^{*}=0.1$.

and anions are increased and decreased, respectively, and thereby $I^{*}$ is increased/decreased if the valence of the cation is higher/lower than that of the anion, or the value of $z_{1}+z_{2}$ is higher/lower than zero.

When $R_{\mathrm{mc}}$ increases, the thickness of the membrane layer shrinks, and correspondingly the strength of the electrical double layer is decreased. For a symmetric electrolyte, that is, the condition for (a), the cation is equal to the anion in absolute valence and $I_{\mathrm{ec}}$ are very close to $I_{\mathrm{ec}}^{0}$. Hence $I^{*}$ is very close to unity, and the variation is trivial when $R_{\mathrm{mc}}$ changes, as Figs. 2, 3 , and 6 show. Owing to richer cations, $I^{*}$ is a little higher than unity, and decreases as $R_{\mathrm{mc}}$ increases, and meanwhile, the strength of the electrical double layer decreases. For the condition when the valence of the cations is higher than that of the anions, as in (b) and (c), since the molar concentration of cations is larger than that in the bulk solution, $I^{*}$ is larger than unity. In this condition, if we raise $R_{\mathrm{mc}}$, due to the decrease in strength of the electrical double layer, the molar concentration of cations is lowered. And therefore $I^{*}$ decreases, as can be seen in Figs. 2, 4, and 7. In contrast, when the valence of the cation is lower than that of the anion, as in (d) and (e), the molar concentration of anions is smaller than that in bulk solution, and hence the scaled electric current is smaller than unity. In this condition, if we raise $R_{\mathrm{mc}}$, due to the decrease in strength of the electrical double layer, the molar concentration of anions is elevated. Therefore, $I^{*}$ increases, as can be seen in Figs. 2, 5, and 8.

The increases in magnitude of both $\rho_{\mathrm{ef}}^{*}$ and $\varepsilon^{*}$ will increase the strength of the electrical double layer in the microchannel, as mentioned in previous work [26]. For the case when the va- 


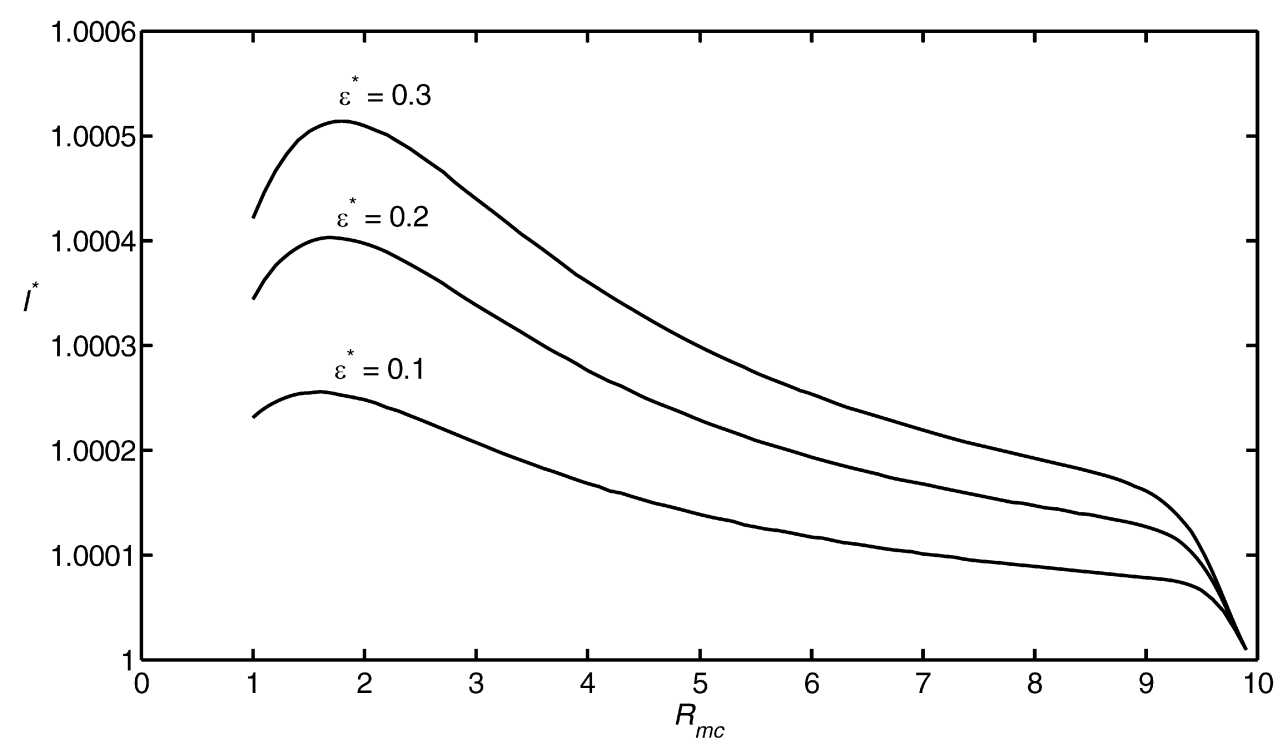

Fig. 6. Variation of $I^{*}$ as a function of $R_{\mathrm{mc}}$ when $\varepsilon^{*}$ changes. $\left(z_{1}, z_{2}\right)=(1,-1),(2,-2)$, and $(3,-3) . \rho_{\mathrm{ef}}^{*}=-0.5$.

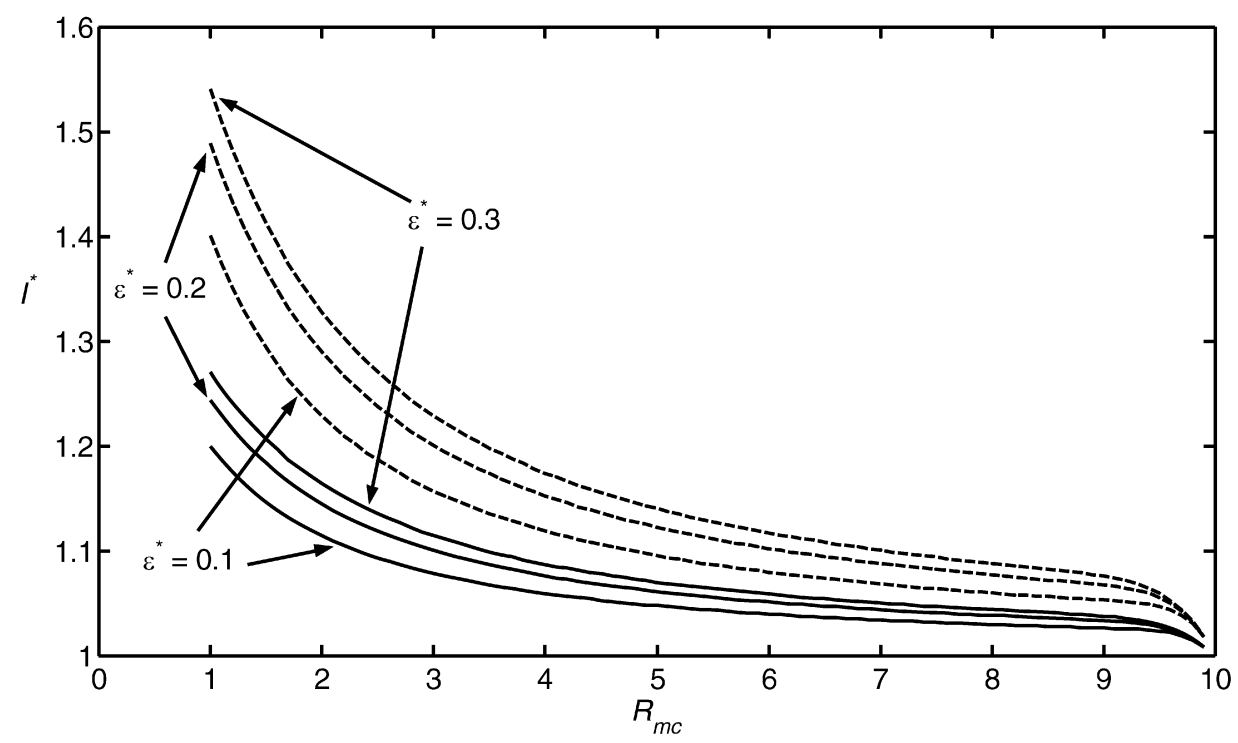

Fig. 7. Variation of $I^{*}$ as a function of $R_{\mathrm{mc}}$ when $\varepsilon^{*}$ changes. Solid curves: $\left(z_{1}, z_{2}\right)=(2,-1)$ and $(3,-2)$. Dashed curves: $\left(z_{1}, z_{2}\right)=(3,-1) . \rho_{\mathrm{ef}}^{*}=-0.5$.

lence of the cation is higher than that of the anion, as in (b) and (c), the increase in magnitude of $\rho_{\mathrm{ef}}^{*}$ or $\varepsilon^{*}$ will elevate $I^{*}$ due to the stronger electrical double layer, as shown in Figs. 4 and 7. Conversely, when the valence of the cation is lower than that of the anion, as in (d) and (e), the increase in magnitude of $\rho_{\mathrm{ef}}^{*}$ or $\varepsilon^{*}$ will lower $I^{*}$, due to a stronger electrical double layer as well, as shown in Figs. 5 and 8.

\section{Conclusions}

The performance of electric-field-assisted membrane modules for the ionic separation efficiency is analyzed. The results show that the effects of system parameters on the ionic separation efficiency are quite different from those on the effluent volumetric flow rate. In the main, the effects of system parameters on ionic separation efficiency are summarized as follows:
(1) For symmetric electrolytes, the effects of system parameters are minimal.

(2) When the valence of the cation is higher than that of the anion, the ionic separation efficiency decreases to unity as the size of the microchannel increases. In addition, an increase either in fixed charge density in the membrane layer or in permittivity of the membrane layer elevates the ionic separation efficiency.

(3) When the valence of the cation is lower than that of the anion, the ionic separation efficiency increases to unity as the size of the microchannel increases. In addition, the increase either in fixed charge density in the membrane layer or in permittivity of the membrane layer lowers the ionic separation efficiency.

(4) The ionic separation efficiency is independent of the strength of the applied electric field. 


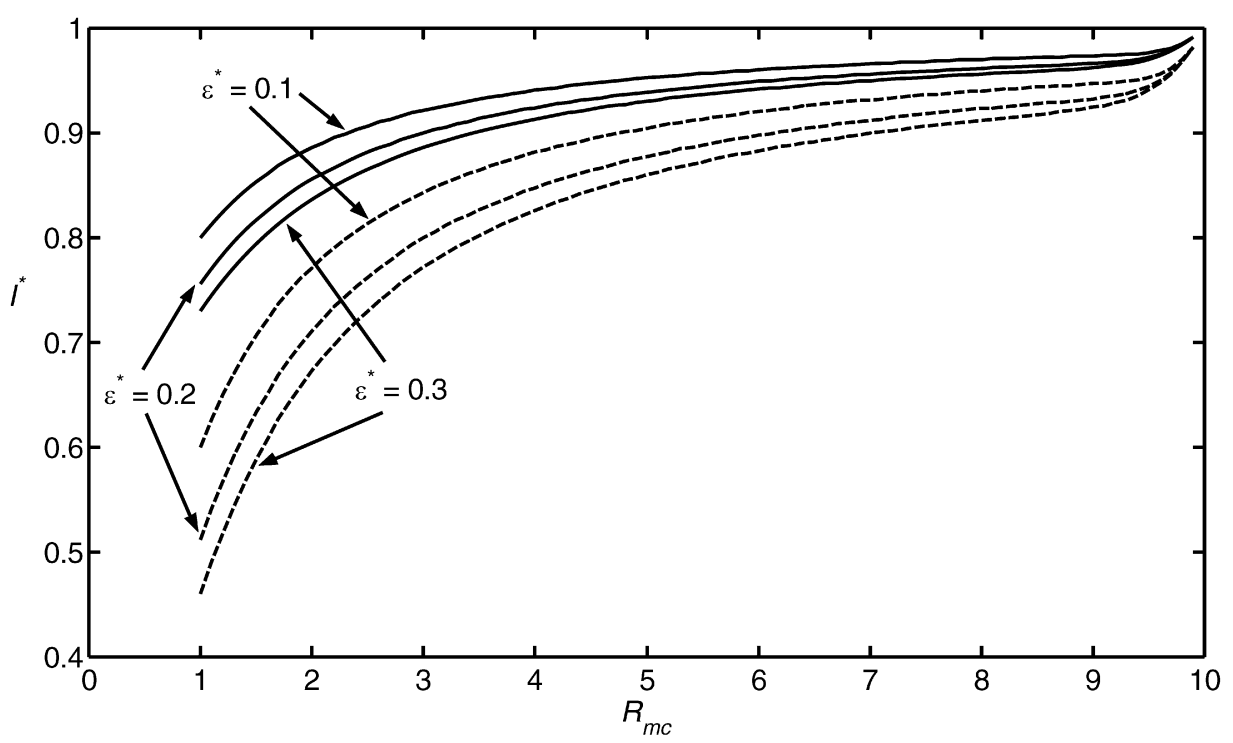

Fig. 8. Variation of $I^{*}$ as a function of $R_{\mathrm{mc}}$ when $\varepsilon^{*}$ changes. Solid curves: $\left(z_{1}, z_{2}\right)=(1,-2)$ and $(2,-3)$. Dashed curves: $\left(z_{1}, z_{2}\right)=(1,-3)$. $\rho_{\mathrm{ef}}^{*}=-0.5$.

\section{Acknowledgments}

This work is supported by the Department of Economics of the Republic of China under Grant 93-EC-17-A-09-S1-019 and the National Science Council of the Republic of China.

\section{Appendix A. Nomenclature}

$A_{1} \quad$ defined in Eqs. (6)-(8)

$c_{i} \quad$ molar concentration of ion $i$

$c_{i}^{0} \quad$ molar concentration of ion $i$ in bulk solution

$E_{z} \quad$ strength of axially applied electric field

$F \quad$ Faraday constant

$i_{z} \quad$ axial electric current density

I ionic strength of electrolyte solution

$I^{*} \quad$ scaled electric current

$I_{0} \quad$ modified Bessel functions of first kind of order 0

$I_{1} \quad$ modified Bessel functions of first kind of order 1

$I_{\mathrm{ec}} \quad$ electric current

$I_{\mathrm{ec}}^{0} \quad$ electric current in the absence of an electrical double layer

$K_{0} \quad$ modified Bessel functions of the second kind of order 0

$K_{1} \quad$ modified Bessel functions of the second kind of order 1

$m \quad$ mobility of ions

$m^{*} \quad$ defined in Eq. (5)

$n \quad$ number of kinds of ions in electrolyte solution

$N_{\mathrm{A}} \quad$ Avogadro's number

$r \quad$ radial coordinate in microchannel unit

$r_{\mathrm{mc}} \quad$ radius of microchannel

$r_{\text {mcu }} \quad$ radius of microchannel unit

$R \quad$ scaled radial coordinate in microchannel unit

$R_{\mathrm{gc}} \quad$ gas constant

$R_{\mathrm{mc}} \quad$ scaled radius of microchannel

$R_{\mathrm{mcu}} \quad$ scaled radius of microchannel unit
$T \quad$ absolute temperature

$u_{i} \quad$ axial velocity of ion $i$

$u_{z} \quad$ axial velocity of electrolyte solution in microchannel

$z \quad$ axial coordinate in microchannel unit

$z_{i} \quad$ valence of ion $i$

$\varepsilon^{*} \quad$ scaled permittivity of membrane layer

$\varepsilon_{\mathrm{mc}} \quad$ permittivity of electrolyte solution

$\varepsilon_{\text {mcs }} \quad$ permittivity of membrane layer

$\kappa \quad$ Debye-Hückel parameter

$\mu \quad$ viscosity of electrolyte solution

$\rho_{\text {ef }} \quad$ fixed charge density in membrane layer

$\rho_{\mathrm{ef}}^{*} \quad$ fixed charge density in membrane layer

$\phi \quad$ electrical potential due to electrical double layer

\section{References}

[1] H. Reiss, I.C. Bassignana, J. Membr. Sci. 11 (1982) 219.

[2] C. Selvey, H. Reiss, J. Membr. Sci. 23 (1985) 11.

[3] J.A. Manzanares, S. Mafe, J. Pellicer, J. Phys. Chem. 95 (1991) 5620.

[4] J.A. Manzanares, W.D. Murphy, S. Mafe, H. Reiss, J. Phys. Chem. 97 (1993) 8524.

[5] V.A. Sokirko, J.A. Manzanares, J. Pellicer, J. Colloid Interface Sci. 168 (1994) 32.

[6] J.P. Hsu, K.L. Yang, J. Phys. Chem. 100 (1996) 12503.

[7] J.P. Hsu, B.T. Liu, J. Phys. Chem. B 101 (1997) 7928.

[8] J.P. Hsu, K.L. Yang, K.C. Ting, Phys. Chem. B 101 (1997) 8984.

[9] J.P. Hsu, B.T. Liu, J. Membr. Sci. 142 (1998) 245.

[10] J.P. Hsu, K.C. Ting, J. Electrochem. Soc. 145 (1998) 1088.

[11] J.P. Hsu, J.M. Jiang, J. Membr. Sci. 158 (1999) 257.

[12] J.P. Hsu, K.C. Ting, Y.H. Shieh, J. Phys. Chem. B 104 (2000) 3492.

[13] C.L. Rice, R. Whitehead, J. Phys. Chem. 69 (1965) 4017.

[14] W.R. Bowen, F. Jenner, J. Colloid Interface Sci. 173 (1995) 388.

[15] G.M. Mala, D. Li, J.D. Dale, Int. J. Heat Mass Transfer 40 (1997) 3079.

[16] G.M. Mala, D. Li, C. Werner, H.J. Jacobasch, Y.B. Ning, Int. J. Heat Fluid Flow 18 (1997) 489.

[17] C. Yang, D. Li, J. Colloid Interface Sci. 194 (1997) 95.

[18] Y. Qian, G. Yang, W.R. Bowen, J. Colloid Interface Sci. 190 (1997) 55.

[19] G.M. Mala, C. Yang, D. Li, Colloids Surf. A 135 (1998) 109. 
[20] C. Yang, D. Li, Colloids Surf. A 143 (1998) 339.

[21] C. Yang, D. Li, J.H. Masliyah, Int. J. Heat Mass Transfer 41 (1998) 4229.

[22] G.M. Mala, D. Li, Int. J. Heat Fluid Flow 20 (1999) 142.

[23] S. Tseng, C.Y. Kao, J.P. Hsu, Electrophoresis 21 (2000) 3541.
[24] J.P. Hsu, C.Y. Kao, J. Phys. Chem. B 105 (2001) 8135.

[25] J.P. Hsu, C.Y. Kao, S. Tseng, C.J. Chen, J. Colloid Interface Sci. 248 (2002) 176.

[26] J.P. Hsu, S.H. Lin, S. Tseng, J. Phys. Chem. B 110 (2006) 10082. 